\title{
Review \\ Stability of Antimicrobials in Elastomeric Pumps: A Systematic Review
}

\author{
Beatriz Fernández-Rubio ${ }^{1}(\mathbb{D})$, Paula del Valle-Moreno ${ }^{1}\left(\mathbb{D}\right.$, Laura Herrera-Hidalgo ${ }^{2, *} \mathbb{D}$, Alicia Gutiérrez-Valencia ${ }^{3}$, \\ Rafael Luque-Márquez ${ }^{4}$, Luis E. López-Cortés ${ }^{5}$ D , José María Gutiérrez-Urbón ${ }^{6} \mathbb{D}$, Sonia Luque-Pardos ${ }^{7}$, \\ Aurora Fernández-Polo ${ }^{8}$ (D) and María V. Gil-Navarro ${ }^{2}$
}

Citation: Fernández-Rubio, B.; del Valle-Moreno, P.; Herrera-Hidalgo, L.; Gutiérrez-Valencia, A.; LuqueMárquez, R.; López-Cortés, L.E.; Gutiérrez-Urbón, J.M.; Luque-Pardos, S.; Fernández-Polo, A.; Gil-Navarro, M.V. Stability of Antimicrobials in Elastomeric Pumps: A Systematic Review. Antibiotics 2022, 11, 45. https://doi.org/10.3390/ antibiotics 11010045

Academic Editor: Marc Maresca

Received: 9 December 2021

Accepted: 28 December 2021

Published: 30 December 2021

Publisher's Note: MDPI stays neutral with regard to jurisdictional claims in published maps and institutional affiliations.

Copyright: (C) 2021 by the authors. Licensee MDPI, Basel, Switzerland. This article is an open access article distributed under the terms and conditions of the Creative Commons Attribution (CC BY) license (https:// creativecommons.org/licenses/by/ $4.0 /)$.
1 Unidad de Gestión Clínica de Farmacia, Hospital Universitario Virgen del Rocío, 41013 Seville, Spain; beatrizfernandezrub@gmail.com (B.F.-R.); pauladelvallem.0876@gmail.com (P.d.V.-M.)

2 Unidad de Gestión Clínica de Farmacia, Instituto de Biomedicina de Sevilla (IBiS), Hospital Universitario Virgen del Rocío, 41013 Seville, Spain; mariav.gil.sspa@juntadeandalucia.es

3 Infección por el VIH y Farmacocinética de Antivirals, Instituto de Biomedicina de Sevilla (IBiS), 41013 Seville, Spain; alicia.gutierrez.valencia@gmail.com

4 Unidad de Gestión Clinica de Enfermedades Infecciosas, Microbiología y Medicina Preventiva, Instituto de Biomedicina de Sevilla (IBiS), Hospital Universitario Virgen del Rocío, 41013 Seville, Spain; rafaeluquemarquez@gmail.com

5 Unidad de Gestión Clínica de Enfermedades Infecciosas, Microbiología y Medicina Preventiva, Instituto de Biomedicina de Sevilla (IBiS), Hospital Universitario Virgen Macarena/CSIC/, 41013 Seville, Spain; luiselopezcortes@gmail.com

6 Unidad de Gestión Clínica de Farmacia, Complexo Hospitalario Universitario de A Coruña, 15006 A Coruna, Spain; Jose.Gutierrez.Urbon@sergas.es

7 Unidad de Gestión Clínica de Farmacia, Hospital del Mar, 08003 Barcelona, Spain; sluque@psmar.cat

8 Servicio de Farmacia, Proa-NEN, Hospital Infantil, Vall d'Hebron Barcelona Hospital Campus, 08035 Barcelona, Spain; aufernan@vhebron.net

* Correspondence: lauraherrerahidalgo@gmail.com; Tel.: +34-955-012-095

Abstract: Outpatient parenteral antimicrobial therapy (OPAThttp) programs have become an important healthcare tool around the world. Portable elastomeric infusion pumps are functional devices for ambulatory delivery of antimicrobial drugs, and their stability is an essential point to guarantee an appropriate infusion administration. We conducted a systematic review to provide a synthesis and a critical evaluation of the current evidence regarding antimicrobial stability in elastomeric pumps. Data sources were PubMed, EMBASE, and Web of Sciences. The review protocol was registered on the Center for Open Science, and it was carried out following the PRISMA guidelines. Studies were eligible if the aim was the evaluation of the physicochemical stability of an antimicrobial agent stored in an elastomeric device. Of the 613 papers identified, 33 met the inclusion criteria. The most studied group of antimicrobials was penicillins, followed by cephalosporins and carbapenems. In general, the stability results of the antimicrobials that have been studied in more than one article agree with each other, with the exception of ampicillin, flucloxacillin, and ceftazidime. The antibiotics that displayed a longer stability were glycopeptides and clindamycin. Regarding the stability of antifungals and antivirals, only caspofungin, voriconazole, and ganciclovir have been investigated. The information provided in this article should be considered in patient treatments within the OPAT setting. Further stability studies are needed to confirm the appropriate use of the antimicrobials included in this program to ensure optimal patient outcomes.

Keywords: antimicrobials; elastomer; OPAT; systematic review

\section{Introduction}

Over the last several decades, outpatient parenteral antimicrobial therapy (OPAT) programs have been implemented as a useful healthcare tool worldwide. These programs enable clinically stable patients to receive optimal antimicrobial treatment after hospital 
discharge [1]. There are two modalities of OPAT programs, one of which involves the administration of the antimicrobial in the patient's home and the other at an infectious disease outpatient center [2]. OPAT programs provide several advantages, such as the improvement of the quality of life of patients, allowing them to continue with their daily routine at home despite receiving intravenous antibiotics. Additionally, they reduce the potential risk of acquiring nosocomial infections or hospital-acquired delirium in elderly patients, and they decrease the mean number of hospitalization days, which is beneficial for the healthcare system [3]. Nevertheless, current OPAT models have some limitations, including the potential administration-related toxicity and the requirement of a healthcare professional in order to set up the pumps [4], especially if the administration is carried out at the outpatient facility.

Portable elastomeric infusion pumps are functional devices for the delivery of intravenous drugs, focused on making the administration easier [5]. They are lightweight devices with a transparent plastic container, inside which there is an elastomeric reservoir, commonly made of latex, polyisoprene, or silicone, which contains the medication. Its operation is based on the elastomeric property of the balloon to release the drug solution at a constant flow along an infusion line [6]. These pumps have undergone significant development, with a rising frequency of use due to the advantages that they offer [7]. The main qualities include portability, non-electronic handling, and ease of management by the patient or a relative, providing more autonomy to the patient. Furthermore, elastomeric pumps allow a safe, continuous drug administration at a constant flow rate and in absolute silence, and they have a better cost effectiveness profile than electronic models [8]. However, the use of elastomeric pumps involves some limitations, including a lower delivery rate accuracy than electronic pumps, the necessity to choose a specific speed through the whole administration, and the lack of an alarm if a failure occurs during the drug delivery. In addition, environmental factors such as external temperature need to be taken into account, as this could affect the stability of antibiotics $[9,10]$.

In this context, antimicrobial stability is an essential point to guarantee an optimal health outcome [11]. Conditions affecting stability are varied, including the concentration of the drug, diluents and additives used; the temperature and duration of the storage; and the composition of the elastomeric device used. Both chemical stability (percentage of the drug that remains after the storage) and physical stability (changes in color or clearness, $\mathrm{pH}$, and particle formation) should be studied. Therefore, stability data are essential requirements for safety management [12,13]. The aim of this systematic review is to provide a synthesis and a critical evaluation of the current evidence regarding antimicrobial stability in elastomeric pumps.

\section{Results}

The initial search found 613 papers after duplicates were removed. Following preliminary title and abstract review, 63 records were selected for full-text review. Thirty papers were excluded because eleven papers were published in a conference abstract format, seven were not contained in an elastomeric device, two measured the stability within blood samples, and ten did not evaluate the physicochemical stability of the antimicrobial. Finally, 33 articles were included in the systematic review (Figure 1).

The principal characteristics of each antimicrobial group, such as drug, reference [14-46], main conditions (composition of the elastomeric device chosen, concentration, diluent, temperature, and duration of storage), chemical and physical stability obtained, and the most relevant comments for the systematic review, are summarized in Tables 1-8. 


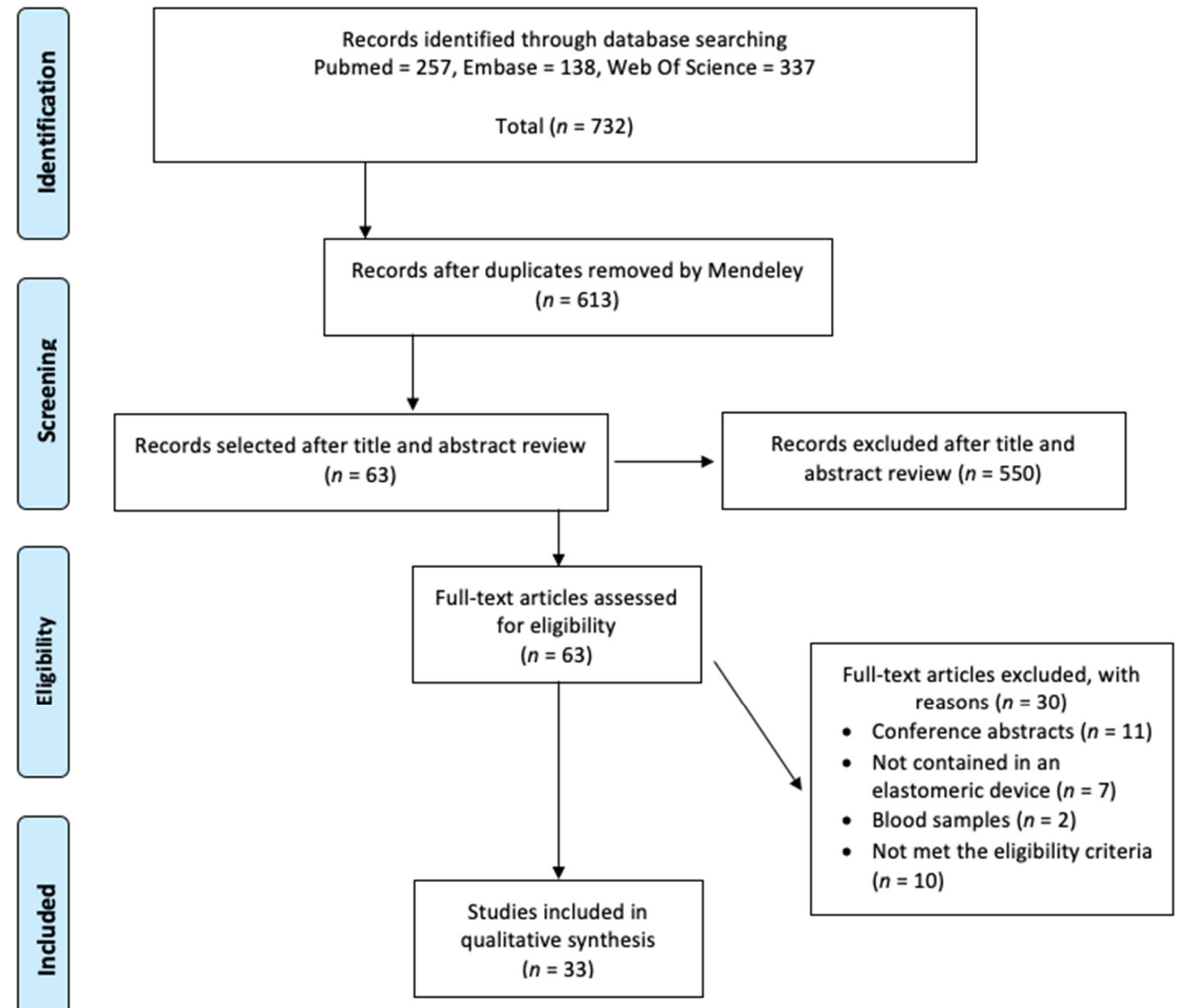

Figure 1. Study selection flowchart.

The most studied group of antimicrobials has been the penicillins. Within the group, amoxicillin, flucloxacillin, and piperacillin/tazobactam have had the most elastomer stability studies performed (Table 1 ). Their maximum stability is just $24 \mathrm{~h}$, though this depends greatly on the temperature, and some exceptions can be found in the case of amoxicillin. Regarding the stability of cephalosporins, cefazolin and ceftazidime are the most commonly used in elastomeric devices, since they are stable in both normal saline and $5 \%$ dextrose in a wide range of concentrations under different storage conditions (Table 2). Additionally, the composition of the elastomeric device can be latex, silicone, or polyisoprene. Meropenem is the most studied carbapenem, including the combination with vaborbactam, a betalactamase inhibitor. In the studies performed with meropenem alone, both experiments proved that the stability is highly dependent on the concentration, since it decreases if the concentration is raised under all storage conditions (Table 3). In relation to aminoglycosides, glycopeptides, and other antibiotics (clindamycin and colistin), their physicochemical stability is variable (Tables 4-6, respectively). With the exception of aminoglycosides, the rest of the antibiotics have shown a long stability contained in elastomeric devices. Regarding the stability of antifungals, just voriconazole and caspofungin have been investigated (Table 7). The stability of caspofungin is longer than that of voriconazole. However, this depends on the composition of the elastomeric device. In relation to antivirals, only ganciclovir was studied in a single paper (Table 8). 
Table 1. Stability of penicillins in elastomeric devices.

\begin{tabular}{|c|c|c|c|c|c|c|c|c|}
\hline Drug & Reference & $\begin{array}{l}\text { Composition of the } \\
\text { Elastomeric Device }\end{array}$ & $\begin{array}{l}\text { Concentration } \\
(\mathrm{mg} / \mathrm{mL})\end{array}$ & Diluent & $\begin{array}{c}\text { Temperature and } \\
\text { Duration of Storage }\end{array}$ & $\begin{array}{l}\text { Chemical } \\
\text { Stability }\end{array}$ & $\begin{array}{l}\text { Physical } \\
\text { Stability }\end{array}$ & Comments \\
\hline \multirow[b]{2}{*}{ Ampicillin } & [14] & Latex & 20 & NS & $\begin{array}{l}5^{\circ} \mathrm{C}: 3 \mathrm{~d} \\
25^{\circ} \mathrm{C}: 8 \mathrm{~h}\end{array}$ & Yes & Not studied & \\
\hline & [15] & Polyisoprene & 50 & $\begin{array}{l}\text { Acetate ringer } \\
\text { solution }\end{array}$ & $\begin{array}{c}4^{\circ} \mathrm{C}: 10 \mathrm{~d} \\
25^{\circ} \mathrm{C}: 24 \mathrm{~h} \\
31.1^{\circ} \mathrm{C}: 24 \mathrm{~h}\end{array}$ & No & Not studied & \\
\hline \multirow{4}{*}{ Amoxicillin } & [16] & Polyisoprene & 25 & NS & $5^{\circ} \mathrm{C}: 48 \mathrm{~h}$ & Yes & Not studied & $\begin{array}{c}\text { Also measured } \\
\text { effectiveness of plasma } \\
\text { amoxicillin } \\
\text { concentrations }\end{array}$ \\
\hline & [17] & Polyisoprene & 20,40 , and 60 & NS & $\begin{array}{l}20^{\circ} \mathrm{C}: 48 \mathrm{~h} \\
25^{\circ} \mathrm{C}: 48 \mathrm{~h}\end{array}$ & $\begin{array}{l}\text { Yes (only at } 20 \text { and } \\
\quad 40 \mathrm{mg} / \mathrm{mL} \text { ) }\end{array}$ & $\begin{array}{c}\text { Yes (not } \\
\text { precipitate or } \\
\text { color changes) }\end{array}$ & $\begin{array}{l}\text { No chemical stability at } \\
\qquad 60 \mathrm{mg} / \mathrm{mL}\end{array}$ \\
\hline & [18] & $\begin{array}{l}\text { Polyisoprene or } \\
\text { silicone }\end{array}$ & $\begin{array}{c}25,50,125, \text { and } \\
250\end{array}$ & Sterile water & $\begin{array}{l}5^{\circ} \mathrm{C}: 24 \mathrm{~h} \\
25^{\circ} \mathrm{C}: 12 \mathrm{~h}\end{array}$ & $\begin{array}{l}\text { Yes (just the } \\
\text { lowest } \\
\text { concentration, } \\
25 \mathrm{mg} / \mathrm{mL} \text { ) }\end{array}$ & Not studied & $\begin{array}{l}\text { No chemical stability at } \\
\text { high concentrations and } \\
\text { at high temperatures } \\
\text { (more than } 30^{\circ} \mathrm{C} \text { ) }\end{array}$ \\
\hline & [19] & Polyisoprene & 25,50 , and 83.3 & NS & $\begin{array}{l}4^{\circ} \mathrm{C}: 48 \mathrm{~h} \\
25^{\circ} \mathrm{C}: 48 \mathrm{~h}\end{array}$ & Yes & Not studied & \\
\hline Benzylpenicillin & [15] & Polyisoprene & 100,000 units $/ \mathrm{mL}$ & $\begin{array}{l}\text { Acetate ringer } \\
\text { solution }\end{array}$ & $\begin{array}{c}4^{\circ} \mathrm{C}: 10 \mathrm{~d} \\
25^{\circ} \mathrm{C}: 24 \mathrm{~h} \\
31.1^{\circ} \mathrm{C}: 24 \mathrm{~h}\end{array}$ & Yes & Not studied & \\
\hline
\end{tabular}


Table 1. Cont.

\begin{tabular}{|c|c|c|c|c|c|c|c|c|}
\hline Drug & Reference & $\begin{array}{l}\text { Composition of the } \\
\text { Elastomeric Device }\end{array}$ & $\begin{array}{l}\text { Concentration } \\
(\mathrm{mg} / \mathrm{mL})\end{array}$ & Diluent & $\begin{array}{c}\text { Temperature and } \\
\text { Duration of Storage }\end{array}$ & $\begin{array}{l}\text { Chemical } \\
\text { Stability }\end{array}$ & $\begin{array}{l}\text { Physical } \\
\text { Stability }\end{array}$ & Comments \\
\hline \multirow{4}{*}{ Flucloxacillin } & [20] & $\begin{array}{l}\text { Silicone and } \\
\text { polyisoprene }\end{array}$ & 10 and 50 & $0.3 \% w / v$ & $\begin{array}{c}5^{\circ} \mathrm{C}: 14 \mathrm{~d} \text {, then } 24 \mathrm{~h} \text { at } \\
32{ }^{\circ} \mathrm{C}\end{array}$ & Yes & Not studied & $\begin{array}{l}\text { Citrate-buffered saline } \\
\text { pH } 7\end{array}$ \\
\hline & {$[21]$} & Polyisoprene & 50 & NS & $\begin{array}{c}5{ }^{\circ} \mathrm{C}: 6 \mathrm{~d} \\
5{ }^{\circ} \mathrm{C}: 6 \mathrm{~d} \text {, then } 24 \mathrm{~h} \text { at } \\
31^{\circ} \mathrm{C}\end{array}$ & Yes & Not studied & $\begin{array}{l}\text { It was not chemically } \\
\text { stable when the } \\
\text { temperature was raised } \\
\text { to } 37^{\circ} \mathrm{C} \text { for } 7 \mathrm{~h} \text { after } 6 \mathrm{~d} \\
\text { at } 5^{\circ} \mathrm{C} \text { and } 24 \mathrm{~h} \text { at } 31^{\circ} \mathrm{C}\end{array}$ \\
\hline & [22] & Polyisoprene & 50 & $\begin{array}{c}\text { NS or } \\
\text { water for injection } \\
\text { with or without } \\
\text { phosphate buffer } \\
\text { (0.384 M; pH 7) }\end{array}$ & $\begin{array}{c}4{ }^{\circ} \mathrm{C}: 6 \mathrm{~d} \\
4{ }^{\circ} \mathrm{C}: 6 \mathrm{~d} \text {, then } 24 \mathrm{~h} \text { at } \\
37^{\circ} \mathrm{C} .\end{array}$ & Yes & Not studied & $\begin{array}{c}\text { At } 37^{\circ} \mathrm{C} \text {, the } \\
\text { unbuffered solution was } \\
\text { not chemically stable }\end{array}$ \\
\hline & [23] & Polyisoprene & 33 & NS & $\begin{array}{l}26.2^{\circ} \mathrm{C}: 24 \mathrm{~h} \\
30.9^{\circ} \mathrm{C}: 24 \mathrm{~h}\end{array}$ & No & Not studied & $\begin{array}{l}\text { Study under real-life } \\
\text { situations }\end{array}$ \\
\hline Mezlocillin & [14] & Latex & 20 & $5 \mathrm{D}$ & $\begin{array}{c}5^{\circ} \mathrm{C}: 7 \mathrm{~d} \\
20^{\circ} \mathrm{C}: 4 \mathrm{w} \\
25^{\circ} \mathrm{C}: 48 \mathrm{~h}\end{array}$ & Yes & Not studied & \\
\hline Nafcillin & [14] & Latex & 20 & NS or 5D & $\begin{array}{c}-20^{\circ} \mathrm{C}: 12 \mathrm{w} \\
5^{\circ} \mathrm{C}: 4 \mathrm{~d} \\
25^{\circ} \mathrm{C}: 24 \mathrm{~h}\end{array}$ & Yes & Not studied & \\
\hline $\begin{array}{l}\text { Penicillin G } \\
\text { sodium }\end{array}$ & [24] & Polyisoprene & $\begin{array}{c}2500 \text { and } 50,000 \\
\text { units } / \mathrm{mL}\end{array}$ & NS or $5 \mathrm{D}$ & $5^{\circ} \mathrm{C}: 21 \mathrm{~d}$ & Yes & $\begin{array}{c}\text { No (pH } \\
\text { consistently } \\
\text { decreased, from } \\
6.4 \text { to } 5.5 ; \text { no } \\
\text { change in } \\
\text { appearance) }\end{array}$ & $\begin{array}{c}\text { After } 28 \mathrm{~d}, 2500 \\
\text { units } / \mathrm{mL} \text { with NS was } \\
\text { not chemically stable }\end{array}$ \\
\hline Piperacillin & [14] & Latex & 30 & NS or $5 \mathrm{D}$ & $\begin{array}{c}-20^{\circ} \mathrm{C}: 4 \mathrm{w} \\
5^{\circ} \mathrm{C}: 7 \mathrm{~d} \\
25^{\circ} \mathrm{C}: 24 \mathrm{~h}\end{array}$ & Yes & Not studied & \\
\hline
\end{tabular}


Table 1. Cont.

\begin{tabular}{|c|c|c|c|c|c|c|c|c|}
\hline Drug & Reference & $\begin{array}{l}\text { Composition of the } \\
\text { Elastomeric Device }\end{array}$ & $\begin{array}{l}\text { Concentration } \\
(\mathrm{mg} / \mathrm{mL})\end{array}$ & Diluent & $\begin{array}{c}\text { Temperature and } \\
\text { Duration of Storage }\end{array}$ & $\begin{array}{l}\text { Chemical } \\
\text { Stability }\end{array}$ & $\begin{array}{l}\text { Physical } \\
\text { Stability }\end{array}$ & Comments \\
\hline \multirow{4}{*}{$\begin{array}{l}\text { Piperacillin/ } \\
\text { tazobactam }\end{array}$} & [25] & Polyisoprene & $67 / 8$ & NS or $5 \mathrm{D}$ & $31.1^{\circ} \mathrm{C}: 24 \mathrm{~h}$ & Yes & Yes $(\mathrm{pH})$ & \\
\hline & [26] & Polyisoprene & $\begin{array}{c}9 / 1.15 \\
50 / 6.2 \\
90 / 11.25\end{array}$ & NS & $35^{\circ} \mathrm{C}: 72 \mathrm{~h}$ & No & $\begin{array}{l}\text { No (pH changed } \\
\text { although not } \\
\text { precipitate or } \\
\text { color changes) }\end{array}$ & \\
\hline & [27] & Polyisoprene & $\begin{array}{c}22 / 3 \\
80 / 10\end{array}$ & NS & $\begin{array}{c}5{ }^{\circ} \mathrm{C}: 13 \mathrm{~d} \text {, then } 24 \mathrm{~h} \text { at } \\
32{ }^{\circ} \mathrm{C}\end{array}$ & Yes & Not studied & $\begin{array}{l}\text { Use of a citrate-buffered } \\
\text { saline diluent } \mathrm{pH} 7\end{array}$ \\
\hline & [23] & Polyisoprene & $50 / 6.25$ & NS & $\begin{array}{l}26.2^{\circ} \mathrm{C}: 24 \mathrm{~h} \\
30.9^{\circ} \mathrm{C}: 24 \mathrm{~h}\end{array}$ & Yes & Not studied & $\begin{array}{l}\text { Study under real-life } \\
\text { situations }\end{array}$ \\
\hline Temocillin & [28] & Polyisoprene & 10 and 20 & Water for injection & $\begin{array}{c}4{ }^{\circ} \mathrm{C}: 4 \mathrm{w} \\
4{ }^{\circ} \mathrm{C}: 4 \mathrm{w} \text {, then } 24 \mathrm{~h} \text { at } \\
25^{\circ} \mathrm{C}\end{array}$ & Yes & Not studied & \\
\hline
\end{tabular}

NS: normal saline, 5D: 5\% dextrose, h: hours, d: days, w: weeks.

Table 2. Stability of cephalosporins in elastomeric devices.

\begin{tabular}{|c|c|c|c|c|c|c|c|c|}
\hline Drug & Reference & $\begin{array}{l}\text { Composition of the } \\
\text { Elastomeric Device }\end{array}$ & $\begin{array}{c}\text { Concentration } \\
(\mathrm{mg} / \mathrm{mL})\end{array}$ & Diluent & $\begin{array}{c}\text { Temperature and } \\
\text { Duration of Storage }\end{array}$ & $\begin{array}{l}\text { Chemical } \\
\text { Stability }\end{array}$ & Physical Stability & Comments \\
\hline \multirow{5}{*}{ Cefazoline } & [14] & Latex & 20 & NS or $5 \mathrm{D}$ & $\begin{array}{c}-20^{\circ} \mathrm{C}: 12 \mathrm{w} \\
5^{\circ} \mathrm{C}: 7 \mathrm{~d} \\
25^{\circ} \mathrm{C}: 24 \mathrm{~h}\end{array}$ & Yes & Not studied & \\
\hline & [25] & Polyisoprene & 25 & NS or $5 \mathrm{D}$ & $31.1^{\circ} \mathrm{C}: 24 \mathrm{~h}$ & Yes & $\begin{array}{l}\text { Yes } \\
(\mathrm{pH})\end{array}$ & \\
\hline & [29] & Polyisoprene & 12.5 and 25 & NS or $5 \mathrm{D}$ & $\begin{array}{l}4^{\circ} \mathrm{C}: 72 \mathrm{~h} \text {, then stored at } \\
35^{\circ} \mathrm{C} \text { for } 12 \mathrm{~h} \text {, followed } \\
\text { by } 25^{\circ} \mathrm{C} \text { for } 12 \mathrm{~h} .\end{array}$ & Yes & $\begin{array}{l}\text { Yes (pH unchanged, } \\
\text { clear/no haziness, no } \\
\text { particles). }\end{array}$ & \\
\hline & [23] & Polyisoprene & 25 & NS & $\begin{array}{l}26.2^{\circ} \mathrm{C}: 24 \mathrm{~h} \\
30.9^{\circ} \mathrm{C}: 24 \mathrm{~h}\end{array}$ & Yes & Not studied & $\begin{array}{c}\text { Study under real-life } \\
\text { situations }\end{array}$ \\
\hline & [30] & Silicone & 5 and 40 & NS or $5 \mathrm{D}$ & $\begin{array}{l}4{ }^{\circ} \mathrm{C}: 26 \mathrm{~d} \\
23^{\circ} \mathrm{C}: 3 \mathrm{~d}\end{array}$ & Yes & Not studied & \\
\hline
\end{tabular}


Table 2. Cont.

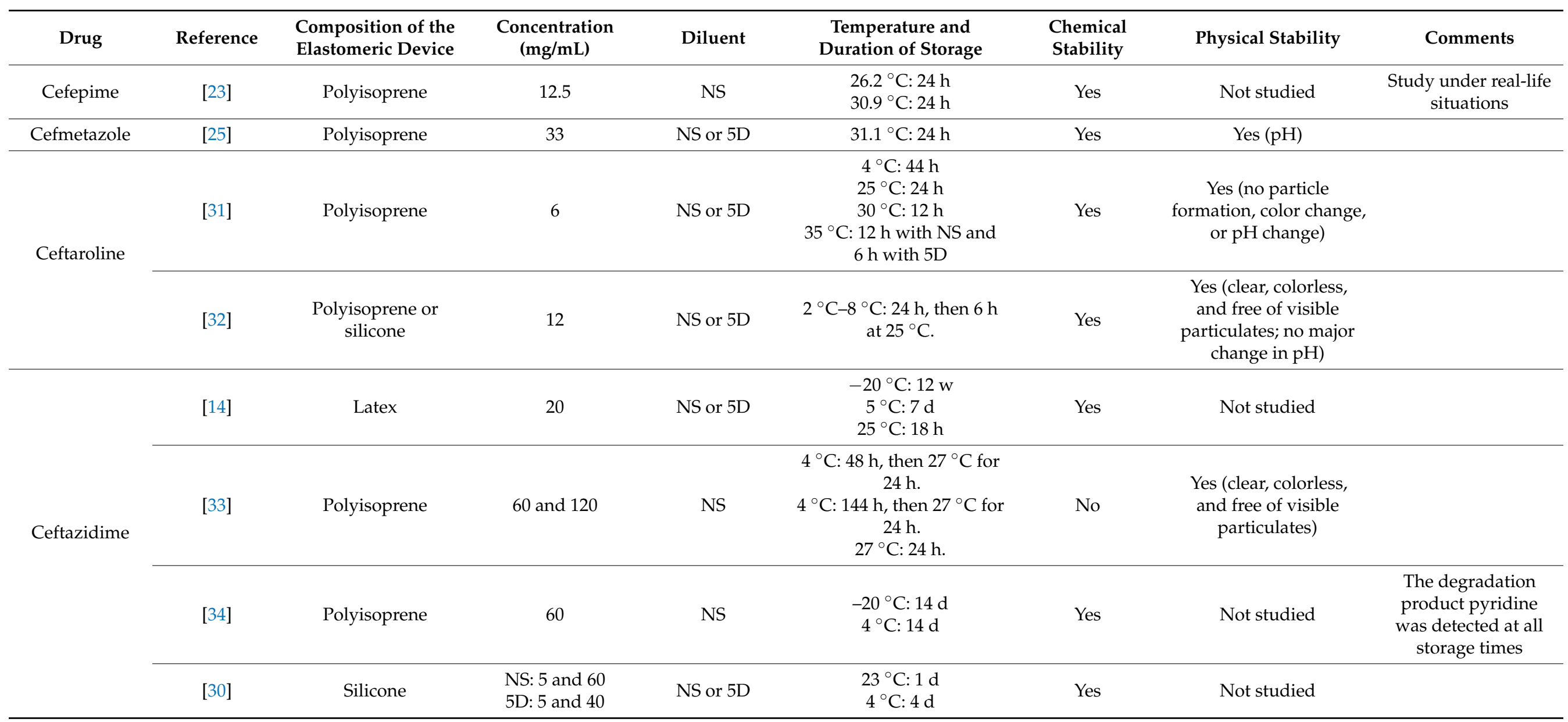


Table 2. Cont.

\begin{tabular}{|c|c|c|c|c|c|c|c|c|}
\hline Drug & Reference & $\begin{array}{l}\text { Composition of the } \\
\text { Elastomeric Device }\end{array}$ & $\begin{array}{l}\text { Concentration } \\
(\mathrm{mg} / \mathrm{mL})\end{array}$ & Diluent & $\begin{array}{c}\text { Temperature and } \\
\text { Duration of Storage }\end{array}$ & $\begin{array}{l}\text { Chemical } \\
\text { Stability }\end{array}$ & Physical Stability & Comments \\
\hline \multirow{2}{*}{$\begin{array}{l}\text { Ceftolozane- } \\
\text { tazobactam }\end{array}$} & [35] & Polyisoprene & $\begin{array}{c}1.25 / 0.63 \\
12.5 / 6.25 \\
25 / 12.5\end{array}$ & NS & $\begin{array}{c}4^{\circ} \mathrm{C}: 7 \mathrm{~d} \\
25^{\circ} \mathrm{C}: 24 \mathrm{~h} \\
37^{\circ} \mathrm{C}: 24 \mathrm{~h}\end{array}$ & Yes & Not studied & $\begin{array}{c}\text { Tazobactam was } \\
\text { more stable than } \\
\text { ceftolozane }\end{array}$ \\
\hline & [36] & Polyisoprene & $\begin{array}{c}1 \mathrm{~g} / 0.5 \mathrm{~g} \\
100 \mathrm{mg} / 50 \mathrm{mg}\end{array}$ & NS or 5D & $\begin{array}{l}5^{\circ} \mathrm{C}: 10 \mathrm{~d} \\
25^{\circ} \mathrm{C}: 24 \mathrm{~h}\end{array}$ & Yes & $\begin{array}{l}\text { Yes (clear and free of } \\
\text { visible particulates; no } \\
\text { changes in } \mathrm{pH} \text { ) }\end{array}$ & \\
\hline \multirow[t]{2}{*}{ Ceftriaxone } & [14] & Latex & 20 & NS or 5D & $\begin{array}{c}-20^{\circ} \mathrm{C}: 26 \mathrm{w} \\
5^{\circ} \mathrm{C}: 10 \mathrm{~d} \\
25^{\circ} \mathrm{C}: 3 \mathrm{~d}\end{array}$ & Yes & Not studied & \\
\hline & [30] & Silicone & 5 and 40 & NS or 5D & $\begin{array}{l}4{ }^{\circ} \mathrm{C}: 14 \mathrm{~d} \\
23^{\circ} \mathrm{C}: 2 \mathrm{~d}\end{array}$ & Yes & Not studied & \\
\hline
\end{tabular}

NS: normal saline, 5D: 5\% dextrose, h: hours, d: days, w: weeks.

Table 3. Stability of carbapenems in elastomeric devices.

\begin{tabular}{|c|c|c|c|c|c|c|c|c|}
\hline Drug & Reference & $\begin{array}{l}\text { Composition of the } \\
\text { Elastomeric Device }\end{array}$ & $\begin{array}{l}\text { Concentration } \\
(\mathrm{mg} / \mathrm{mL})\end{array}$ & Diluent & $\begin{array}{c}\text { Temperature and } \\
\text { Duration of Storage }\end{array}$ & Chemical Stability & Physical Stability & Comments \\
\hline \multirow[b]{2}{*}{ Doripenem } & [25] & Polyisoprene & 12.5 & NS or $5 \mathrm{D}$ & $31.1^{\circ} \mathrm{C}: 24 \mathrm{~h}$ & No & Yes $(\mathrm{pH})$ & \\
\hline & [37] & Polyisoprene & 5 and 10 & NS or $5 \mathrm{D}$ & $\begin{array}{c}-20{ }^{\circ} \mathrm{C}: 28 \mathrm{~d} \\
4{ }^{\circ} \mathrm{C}: 10 \mathrm{~d} \text { in NS and } \\
7 \mathrm{~d} \text { in } 5 \mathrm{~d} \\
25^{\circ} \mathrm{C}: 24 \mathrm{~h} \text { in NS and } \\
16 \mathrm{~h} \text { in } 5 \mathrm{D}\end{array}$ & Yes & No & $\begin{array}{l}\text { A white precipitate, } \\
\text { which returned to } \\
\text { solution by shaking, was } \\
\text { noted after thawing the } \\
\text { frozen containers }\end{array}$ \\
\hline Ertapenem & [38] & Polyisoprene & 10 & NS & $5^{\circ} \mathrm{C}: 72 \mathrm{~h}$ & Yes & $\begin{array}{c}\text { Not } \\
\text { studied }\end{array}$ & \\
\hline $\begin{array}{l}\text { Imipenem- } \\
\text { cilastatin }\end{array}$ & [14] & Latex & 5 & NS or 5D & $\begin{array}{l}5^{\circ} \mathrm{C}: 1 \mathrm{~d} \\
25^{\circ} \mathrm{C}: 4 \mathrm{~h}\end{array}$ & Yes & $\begin{array}{c}\text { Not } \\
\text { studied }\end{array}$ & \\
\hline
\end{tabular}


Table 3. Cont.

\begin{tabular}{|c|c|c|c|c|c|c|c|c|}
\hline Drug & Reference & $\begin{array}{l}\text { Composition of the } \\
\text { Elastomeric Device }\end{array}$ & $\begin{array}{l}\text { Concentration } \\
(\mathrm{mg} / \mathrm{mL})\end{array}$ & Diluent & $\begin{array}{c}\text { Temperature and } \\
\text { Duration of Storage }\end{array}$ & Chemical Stability & Physical Stability & Comments \\
\hline \multirow{3}{*}{ Meropenem } & [25] & Polyisoprene & 12.5 & NS or $5 \mathrm{D}$ & $31.1^{\circ} \mathrm{C}: 24 \mathrm{~h}$ & No & Yes $(\mathrm{pH})$ & \\
\hline & [39] & Polyisoprene & $6,12,20$, and 25 & NS & $\begin{array}{c}5{ }^{\circ} \mathrm{C}: 6 \mathrm{~d} \\
5{ }^{\circ} \mathrm{C}: 48 \mathrm{~h}, \text { then } 4 \mathrm{~d} \text { at } \\
25^{\circ} \mathrm{C}\end{array}$ & $\begin{array}{l}\text { Yes (just the lowest } \\
\text { concentration, } \\
6 \mathrm{mg} / \mathrm{mL} \text { ) }\end{array}$ & Yes $(\mathrm{pH})$ & $\begin{array}{c}\text { At higher concentrations } \\
(25 \mathrm{mg} / \mathrm{mL}) \text {, no chemical } \\
\text { stability }\end{array}$ \\
\hline & {$[40]$} & Polyisoprene & 4,10, and 20 & NS & $5^{\circ} \mathrm{C}: 5 \mathrm{~d}$ & Yes & $\begin{array}{c}\text { Not } \\
\text { studied }\end{array}$ & $\begin{array}{l}\text { The lowest concentration } \\
(4 \mathrm{mg} / \mathrm{mL}) \text { showed } \\
\text { chemical stability for } 7 \mathrm{~d}\end{array}$ \\
\hline $\begin{array}{l}\text { Meropenem/ } \\
\text { vaborbactam }\end{array}$ & [41] & Polyisoprene & $5.7 / 5.7$ & NS & $\begin{array}{l}4^{\circ} \mathrm{C}: 120 \mathrm{~h} \\
24^{\circ} \mathrm{C}: 12 \mathrm{~h}\end{array}$ & Yes & $\begin{array}{c}\text { Not } \\
\text { studied }\end{array}$ & \\
\hline
\end{tabular}

NS: normal saline, 5D: 5\% dextrose, h: hours, d: days.

Table 4. Stability of aminoglycosides in elastomeric devices.

\begin{tabular}{|c|c|c|c|c|c|c|c|c|}
\hline Drug & Reference & $\begin{array}{l}\text { Composition of the } \\
\text { Elastomeric Device }\end{array}$ & $\begin{array}{l}\text { Concentration } \\
(\mathrm{mg} / \mathrm{mL})\end{array}$ & Diluent & $\begin{array}{c}\text { Temperature and } \\
\text { Duration of Storage }\end{array}$ & $\begin{array}{l}\text { Chemical } \\
\text { Stability }\end{array}$ & Physical Stability & Comments \\
\hline Gentamicin & [14] & Latex & 0.8 & NS & $25^{\circ} \mathrm{C}: 24 \mathrm{~h}$ & Yes & Not studied & \\
\hline Tobramycin & [14] & Latex & 0.8 & NS & $25^{\circ} \mathrm{C}: 24 \mathrm{~h}$ & Yes & Not studied & \\
\hline
\end{tabular}

Table 5. Stability of glycopeptides in elastomeric devices.

\begin{tabular}{|c|c|c|c|c|c|c|c|c|}
\hline Drug & Reference & $\begin{array}{l}\text { Composition of the } \\
\text { Elastomeric Device }\end{array}$ & $\begin{array}{l}\text { Concentration } \\
(\mathrm{mg} / \mathrm{mL})\end{array}$ & Diluent & $\begin{array}{c}\text { Temperature and } \\
\text { Duration of Storage }\end{array}$ & $\begin{array}{l}\text { Chemical } \\
\text { Stability }\end{array}$ & $\begin{array}{l}\text { Physical } \\
\text { Stability }\end{array}$ & Comments \\
\hline Telavancin & [42] & Polyisoprene & 0.6 and 8.0 & $\begin{array}{c}\text { NS, 5D, } \\
\text { orsterilized } \\
\text { water }\end{array}$ & $5^{\circ} \mathrm{C}: 8 \mathrm{~d}$ & Yes & Yes (pH) & $\begin{array}{l}\text { Sterilized water }(0.6 \mathrm{mg} / \mathrm{mL}) \text { and } \\
\text { NS }(8.0 \mathrm{mg} / \mathrm{mL}) \text { were followed } \\
\text { by Ringer's lactate solution }\end{array}$ \\
\hline Vancomycin & [14] & Latex & 5 & NS or $5 \mathrm{D}$ & $\begin{array}{l}-20^{\circ} \mathrm{C}: 9 \mathrm{w} \\
5{ }^{\circ} \mathrm{C}: 14 \mathrm{~d} \\
25^{\circ} \mathrm{C}: 24 \mathrm{~h}\end{array}$ & Yes & Not studied & \\
\hline
\end{tabular}

NS: normal saline, 5D: 5\% dextrose, h: hours, d: days, w: weeks. 
Table 6. Stability of other antibiotics in elastomeric devices.

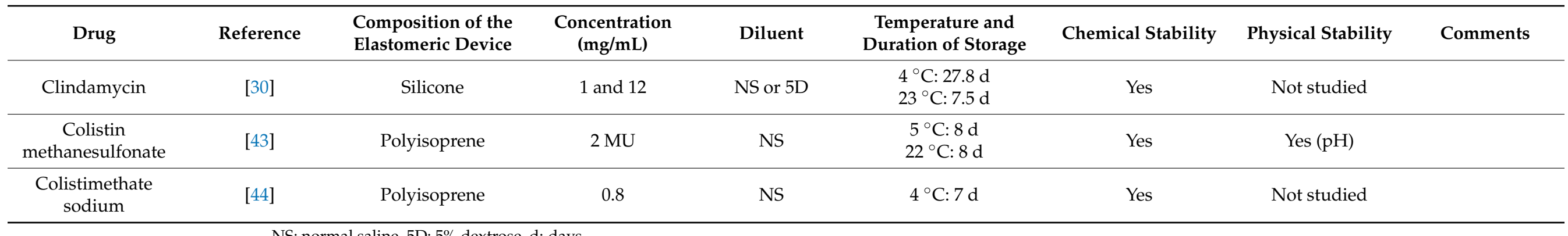

NS: normal saline, 5D: 5\% dextrose, d: days.

Table 7. Stability of antifungals in elastomeric devices.

\begin{tabular}{|c|c|c|c|c|c|c|c|c|}
\hline Drug & Reference & $\begin{array}{l}\text { Composition of the } \\
\text { Elastomeric Device }\end{array}$ & $\begin{array}{l}\text { Concentration } \\
(\mathrm{mg} / \mathrm{mL})\end{array}$ & Diluent & $\begin{array}{c}\text { Temperature and } \\
\text { Duration of Storage }\end{array}$ & Chemical Stability & Physical Stability & Comments \\
\hline Caspofungin & [45] & $\begin{array}{l}\text { Polyisoprene or } \\
\text { silicone }\end{array}$ & $0.2,0.28$, and 0.5 & NS & $\begin{array}{l}5^{\circ} \mathrm{C}: 14 \mathrm{~d} \\
25^{\circ} \mathrm{C}: 60 \mathrm{~h}\end{array}$ & $\begin{array}{l}\text { Yes, in the } \\
\text { polyisoprene infuser }\end{array}$ & $\begin{array}{l}\text { Not } \\
\text { studied }\end{array}$ & $\begin{array}{l}\text { Not chemically } \\
\text { stable in the } \\
\text { silicone infuser }\end{array}$ \\
\hline Voriconazol & [46] & Polyisoprene & 2 & NS or $5 \mathrm{D}$ & $\begin{array}{l}4{ }^{\circ} \mathrm{C}: 96 \mathrm{~h} \\
25^{\circ} \mathrm{C}: 4 \mathrm{~h} \\
35^{\circ} \mathrm{C}: 4 \mathrm{~h}\end{array}$ & Yes & Not studied & \\
\hline
\end{tabular}

NS: normal saline, 5D: 5\% dextrose, h: hours, d: days.

Table 8. Stability of antivirals in elastomeric devices.

\begin{tabular}{|c|c|c|c|c|c|c|c|c|}
\hline Drug & Reference & $\begin{array}{l}\text { Composition of the } \\
\text { Elastomeric Device }\end{array}$ & $\begin{array}{c}\text { Concentration } \\
(\mathrm{mg} / \mathrm{mL})\end{array}$ & Diluent & $\begin{array}{c}\text { Temperature and } \\
\text { Duration of Storage }\end{array}$ & Chemical Stability & Physical Stability & Comments \\
\hline Ganciclovir & [14] & Latex & 5 & NS & $\begin{array}{c}5^{\circ} \mathrm{C}: 5 \mathrm{~d} \\
25^{\circ} \mathrm{C}: 24 \mathrm{~h}\end{array}$ & Yes & Not studied & \\
\hline
\end{tabular}

NS: normal saline, h: hours, d: days. 


\section{Discussion}

Considering that elastomeric devices have shown their great utility in OPAT [47], this systematic review provides a comprehensive overview of antimicrobial stability, both physical and chemical, in elastomeric pumps.

Stability in elastomers has been mainly studied in antibiotics, compared to the experiments published of antivirals and antifungals. Penicillins (in particular amoxicillin, flucloxacillin, and piperacillin/tazobactam) and cephalosporins (especially ceftazidime) are the groups of antibiotics that stand out. In general, the stability results of the antimicrobials that have been studied in more than one article agree with each other. However, some exceptions can be found in the case of ampicillin [14,15], flucoxacillin [20-23], and ceftazidime [14,30,33,34]. Regarding ampicillin, the conditions for each of the experiments were different in terms of their elastomer composition, concentration, and diluent. Therefore, it is difficult to draw firm conclusions. In the case of ceftazidime, results also differ between the studies, but chemical instability of this antibiotic seems to occur when the concentration is high $(120 \mathrm{mg} / \mathrm{mL})$. On the contrary, a lack of stability of flucloxacillin in elastomers is related to high temperatures. Additionally, it should be remarked that, while chemical stability is reflected in the results of all the studies, physical stability is only included in a few of them, with the changes in $\mathrm{pH}$ and color being the most studied.

The composition of the elastomeric reservoir varied between the studies, although the most prevalent has been polyisoprene, followed by latex and silicone. Polyisoprene is a synthetic polymer that provides many of the same properties as natural rubber latex without the latex allergen concerns. Both polymers offer good resistance to most alcohols, acids, bases, and polar solvents and are well-suited for low-temperature environments. In relation to silicone, it is a material that is non-reactive, stable, and resistant to extreme environments and temperatures while still maintaining its useful properties $[48,49]$. There are no published studies that establish a relationship between the material of the elastomeric pump and drug stability. However, one of the studies included in the review has found that, depending on the polymer used, the stability varies [45]. Therefore, it is a factor to take into account in future stability studies. Additionally, it should be noted that, in the case of widely used antibiotics such as vancomycin [14,30] or ceftriaxone [14,30], no published study has analyzed their stability in polyisoprene elastomers. Furthermore, some antimicrobials useful in the OPAT setting have not been studied contained in elastomeric devices. Among these antimicrobials, teicoplanin is particularly relevant, since glycopeptides, along with beta lactams, are the most frequently used antibiotics in OPAT programs [50]. Furthermore, there is a lack of stability studies in elastomers of antimicrobials such as daptomycin [51,52] or amphotericin $b[53,54]$, although this antifungal, along with fluconazole, anidulafungin and micafungin, is used in OPAT programs.

The main strength of this analysis is that it is the first systematic dealing with the physical and chemical stability of antimicrobials in elastomeric devices. Recently, two reviews related to the stability of antimicrobials in elastomers have been published. The first one [55] updates the data from a previous study [56] that was carried out to find out if the stability of antimicrobials in elastomeric pumps meets the quality standards of the Yellow Covered Document (YCD) from the UK National Health System. The second one [57] focuses on evaluating the different means of using elastomeric infusion pumps in out-of-hospital administration of intravenous antibiotics. Therefore, there is no systematic review that provides information on the physicochemical stability of antimicrobials in elastomers together.

This systematic review has some limitations: the analytical techniques used in the different studies have not been described, so there could be certain differences in the methodology of the experiments that have not been analyzed. In addition, this study was limited to including only peer-reviewed articles, so there may be further gray literature supporting the stability of some of the drugs mentioned. 


\section{Materials and Methods}

The review protocol was registered on the Center for Open Science (DOI number: 10.17605/OSF.IO/Y2KJV), and it was carried out following the main criteria of the Preferred Reporting Items for Systematic Reviews and Meta-Analyses (PRISMA) Equity 2012 Extension declaration [58].

\subsection{Eligibility Criteria}

We selected the studies that met the following inclusion criteria:

1. The study drug was an antimicrobial agent, including antibiotics, antivirals, and antifungals.

2. The aim of the study was the evaluation of the physicochemical stability of the antimicrobial agent stored in an elastomeric device.

We dismissed the studies that met the following exclusion criteria:

3. Studies that evaluated admixtures of multiple drugs in the same solution.

4. Studies that measured the serum disposition of the antimicrobial agent in patients.

5. Conference abstracts.

\subsection{Information Sources}

An electronic literature search was performed using MEDLINE (through PubMed interface), EMBASE, and Web of Science Core Collection databases on 1 July 2020, with no publication date restrictions. The search strategy was composed of MeSH terms and free text (keywords and synonyms) combined with Boolean operators. The search strategy was arranged according to each database. Additionally, the reference lists of selected studies were hand-searched to identify any other relevant studies. The search strategy is detailed in Table 9.

Table 9. Complete search strategy for different databases.

\begin{tabular}{cl}
\hline Healthcare Database & \multicolumn{1}{c}{ Search Strategy } \\
\hline PubMed & $\begin{array}{l}\text { (stability) AND (elastomer OR elastomeric) AND (anti-infective agent } \\
\text { OR antibiotic OR antimicrobial) }\end{array}$ \\
\hline EMBASE & $\begin{array}{l}\text { ('stability'/exp) AND ('elastomer'/exp OR 'elastomeric' / exp) AND } \\
\text { ('anti-infective agent' / exp OR 'antibiotic' / exp OR } \\
\text { 'antimicrobial'/exp) }\end{array}$ \\
\hline Web of Science & $\begin{array}{l}\text { TS = (stability AND (elastomer OR elastomeric) AND (anti-infective } \\
\text { agent OR antibiotic OR antimicrobial)) }\end{array}$ \\
\hline
\end{tabular}

\subsection{Study Selection}

Two independent reviewers (B.F.-R. and P.d.V.-M.) screened the titles and abstracts of all eligible publications for possible inclusion after duplicate removal. To ensure interrater reliability, $100 \%$ of the articles were assessed independently by both authors. The articles included were read at their full length before a final decision on inclusion. Any disagreement was settled by consensus with a third reviewer (L.H.-H.).

\subsection{Data Collection and Analysis}

Two reviewers (B.F.-R. and P.d.V.-M.) independently extracted data, and L.H.-H. examined all extraction sheets to ensure their accuracy. We explicitly stated whether there were any data missing from the studies. For each publication, the following variables were registered:

1. Antimicrobial drug studied.

2. Author details and year of publication.

3. Conditions:

Composition of the elastomeric device used. 


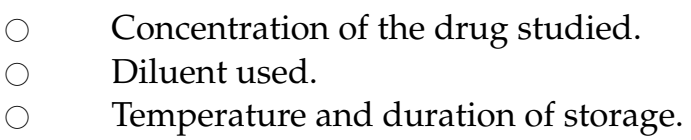

4. Chemical stability demonstrated under each condition: concentration of all samples remained higher than $90 \%$ of the original concentration.

5. Physical stability demonstrated under each condition: particle formation, changes in color or clearness, and $\mathrm{pH}$ analysis.

6. Comments, included buffers of other additives used.

\section{Conclusions}

The information provided in this systematic review should be considered in the treatment of patient within the OPAT setting. Nevertheless, further stability studies are needed to confirm the appropriate use of the antimicrobials included in this program to ensure optimal patient outcomes.

Author Contributions: B.F.-R. wrote the manuscript; B.F.-R. and P.d.V.-M. conducted the research and analyzed the data. L.H.-H. and M.V.G.-N. supervised the project. A.G.-V.; R.L.-M.; L.E.L.-C.; J.M.G.-U.; S.L.-P. and A.F.-P. reviewed and contributed to the final manuscript. All authors have read and agreed to the published version of the manuscript.

Funding: This work was supported by the Sociedad Española de Farmacia Hospitalaria and the AFinf Working Group for the project "Stability study of antimicrobials under conditions analogous to the outpatient parenteral antibiotic therapy program (OPAT)". A.G.-V. was supported by the Instituto de Salud Carlos III, co-financed by the European Development Regional Fund ("A way to achieve Europe"), Subprograma Miguel Servet (grant CP19/00159). L.H.-H. was supported by the Instituto de Salud Carlos III, Subprograma Rio Hortega (grant CM19/00152).

Conflicts of Interest: The authors declare no conflict of interest.

\section{References}

1. Paladino, J.A.; Poretz, D. Outpatient Parenteral Antimicrobial Therapy Today. Clin. Infect. Dis. 2010, 51, S198-S208. [CrossRef] [PubMed]

2. Hamad, Y.; Dodda, S.; Frank, A.; Beggs, J.; Sleckman, C.; Kleinschmidt, G.; Lane, M.A.; Burnett, Y. Perspectives of Patients on Outpatient Parenteral Antimicrobial Therapy: Experiences and Adherence. Open Forum Infect. Dis. 2020, 7, ofaa205. [CrossRef] [PubMed]

3. Mitchell, E.D.; Czoski Murray, C.; Meads, D.; Minton, J.; Wright, J.; Twiddy, M. Clinical and Cost-Effectiveness, Safety and Acceptability of Community Intravenous Antibiotic Service Models: CIVAS Systematic Review. BMJ Open 2017, 7, e013560. [CrossRef]

4. Muldoon, E.G.; Snydman, D.R.; Penland, E.C.; Allison, G.M. Are We Ready for an Outpatient Parenteral Antimicrobial Therapy Bundle? A Critical Appraisal of the Evidence. Clin. Infect. Dis. 2013, 57, 419-424. [CrossRef]

5. Bugeja, S.J.; Stewart, D.; Vosper, H. Clinical Benefits and Costs of an Outpatient Parenteral Antimicrobial Therapy Service. Res. Soc. Adm. Pharm. 2021, 17, 1758-1763. [CrossRef]

6. Diamantis, S.; Longuet, P.; Lesprit, P.; Gauzit, R. Terms of Use of Outpatient Parenteral Antibiotic Therapy. Infect. Dis. Now 2021, 51, 14-38. [CrossRef]

7. Hobbs, J.G.; Ryan, M.K.; Ritchie, B.; Sluggett, J.K.; Sluggett, A.J.; Ralton, L.; Reynolds, K.J. Protocol for a Randomised Crossover Trial to Evaluate Patient and Nurse Satisfaction with Electronic and Elastomeric Portable Infusion Pumps for the Continuous Administration of Antibiotic Therapy in the Home: The Comparing Home Infusion Devices (CHID) Study. BMJ Open 2017, 7, e016763. [CrossRef] [PubMed]

8. Abe, T.; Matsuzaka, K.; Nakayama, T.; Otsuka, M.; Sagara, A.; Sato, F.; Yumoto, T. Impact of Air Temperature and Drug Concentration on Liquid Emission from Elastomeric Pumps. J. Pharm. Health Care Sci. 2021, 7, 1. [CrossRef]

9. Mohseni, M.; Ebneshahidi, A. The Flow Rate Accuracy of Elastomeric Infusion Pumps After Repeated Filling. Anesth. Pain Med. 2014, 4, e14989. [CrossRef] [PubMed]

10. Remerand, F.; Vuitton, A.S.; Palud, M.; Buchet, S.; Pourrat, X.; Baud, A.; Laffon, M.; Fusciardi, J. Elastomeric Pump Reliability in Postoperative Regional Anesthesia: A Survey of 430 Consecutive Devices. Anesth. Analg. 2008, 107, 2079-2084. [CrossRef] [PubMed]

11. Chapman, A.L.N.; Patel, S.; Horner, C.; Gilchrist, M.; Seaton, R.A. Outpatient Parenteral Antimicrobial Therapy: Updated Recommendations from the UK. J. Antimicrob. Chemother. 2019, 74, 3125-3127. [CrossRef] [PubMed] 
12. Perks, S.J.; Lanskey, C.; Robinson, N.; Pain, T.; Franklin, R. Systematic Review of Stability Data Pertaining to Selected Antibiotics Used for Extended Infusions in Outpatient Parenteral Antimicrobial Therapy (OPAT) at Standard Room Temperature and in Warmer Climates. Eur. J. Hosp. Pharm. 2020, 27, 65-72. [CrossRef] [PubMed]

13. Seaton, R.A.; Barr, D.A. Outpatient Parenteral Antibiotic Therapy: Principles and Practice. Eur. J. Intern. Med. 2013, 24, 617-623. [CrossRef] [PubMed]

14. Allen, L.V.; Stiles, M.L.; Prince, S.J.; Smeeding, J. Stability of 14 Drugs in the Latex Reservoir of an Elastomeric Infusion Device. Am. J. Health Syst. Pharm. 1996, 53, 2740-2743. [CrossRef] [PubMed]

15. Nakamura, T.; Enoki, Y.; Uno, S.; Uwamino, Y.; Iketani, O.; Hasegawa, N.; Matsumoto, K. Stability of Benzylpenicillin Potassium and Ampicillin in an Elastomeric Infusion Pump. J. Infect. Chemother. 2018, 24, 856-859. [CrossRef] [PubMed]

16. Arensdorff, L.; Boillat-Blanco, N.; Decosterd, L.; Buclin, T.; de Vallière, S. Adequate Plasma Drug Concentrations Suggest That Amoxicillin Can Be Administered by Continuous Infusion Using Elastomeric Pumps. J. Antimicrob. Chemother. 2017, 72, $2613-2615$. [CrossRef] [PubMed]

17. Arlicot, N.; Marie, A.; Cade, C.; Laffon, M.; Antier, D. Stability of Amoxicillin in Portable Pumps Is Drug Concentration Dependent. Pharmazie 2011, 66, 631-632.

18. Binson, G.; Grignon, C.; Le Moal, G.; Lazaro, P.; Lelong, J.; Roblot, F.; Venisse, N.; Dupuis, A. Overcoming Stability Challenges during Continuous Intravenous Administration of High-Dose Amoxicillin Using Portable Elastomeric Pumps. PLoS ONE 2019, 14, e0221391. [CrossRef]

19. Garg, S.; Kauffmann, K.; Othman, A.; Ticehurst, R. Stability assessment of extemporaneous formulation of amoxicillin for parenteral antimicrobial therapy. Curr. Pharm. Anal. 2012, 8, 375-380. [CrossRef]

20. Allwood, M.C.; Stonkute, D.; Wallace, A.; Wilkinson, A.-S.; Hills, T.; Jamieson, C. BSAC Drug Stability Working Party Assessment of the Stability of Citrate-Buffered Flucloxacillin for Injection When Stored in Two Commercially Available Ambulatory Elastomeric Devices: INfusor LV (Baxter) and Accufuser (Woo Young Medical): A Study Compliant with the NHS Yellow Cover Document (YCD) Requirements. Eur. J. Hosp. Pharm. 2020, 27, 90-94. [CrossRef]

21. Carroll, J.A. Stability of Flucloxacillin in Elastomeric Infusion Devices. J. Pharm. Pract. Res. 2005, 35, 90-93. [CrossRef]

22. To, T.-P.; Ching, M.S.; Ellis, A.G.; Williams, L.; Garrett, M.K. Stability of Intravenous Flucloxacillin Solutions Used for Hospital-inthe-Home. J. Pharm. Pract. Res. 2010, 40, 101-105. [CrossRef]

23. Voumard, R.; Van Neyghem, N.; Cochet, C.; Gardiol, C.; Decosterd, L.; Buclin, T.; de Valliere, S. Antibiotic Stability Related to Temperature Variations in Elastomeric Pumps Used for Outpatient Parenteral Antimicrobial Therapy (OPAT). J. Antimicrob. Chemother. 2017, 72, 1462-1465. [CrossRef] [PubMed]

24. Hossain, M.A.; Friciu, M.; Aubin, S.; Leclair, G. Stability of Penicillin G Sodium Diluted with 0.9\% Sodium Chloride Injection or 5\% Dextrose Injection and Stored in Polyvinyl Chloride Bag Containers and Elastomeric Pump Containers. Am. J. Health Syst. Pharm. 2014, 71, 669-673. [CrossRef] [PubMed]

25. Akahane, M.; Enoki, Y.; Saiki, R.; Hayashi, Y.; Hiraoka, K.; Honma, K.; Itagaki, M.; Gotoda, M.; Shinoda, K.; Hanyu, S.; et al. Stability of Antimicrobial Agents in an Elastomeric Infusion Pump Used for Outpatient Parenteral Antimicrobial Therapy. Int. J. Infect. Dis. 2021, 103, 464-468. [CrossRef] [PubMed]

26. De Calbiac, P.; Lamoureux, F.; Pourrat, X.; Bretault, L.; Marchand, S.; Grassin, J.; Antier, D. Treatment of bronchial superinfections: Data related to stability of antibiotics in portable pumps. Therapie 2006, 61, 139-144. [PubMed]

27. Jamieson, C.; Ozolina, L.; Seaton, R.A.; Gilchrist, M.; Hills, T.; Drummond, F.; Wilkinson, A.S.; BSAC Drug Stability Testing Working Group. Assessment of the Stability of Citrate-Buffered Piperacillin/Tazobactam for Continuous Infusion When Stored in Two Commercially Available Elastomeric Devices for Outpatient Parenteral Antimicrobial Chemotherapy: A Study Compliant with the NHS Yellow Cover Document Requirements. Eur. J. Hosp. Pharm. 2020. [CrossRef]

28. Carryn, S.; Couwenbergh, N.; Tulkens, P.M. Long-Term Stability of Temocillin in Elastomeric Pumps for Outpatient Antibiotic Therapy in Cystic Fibrosis Patients. J. Antimicrob. Chemother. 2010, 65, 2045-2046. [CrossRef] [PubMed]

29. Patel, R.P.; Jacob, J.; Sedeeq, M.; Ming, L.C.; Wanandy, T.; Zaidi, S.T.R.; Peterson, G.M. Stability of Cefazolin in Polyisoprene Elastomeric Infusion Devices. Clin. Ther. 2018, 40, 664-667. [CrossRef]

30. Walker, S.E.; Iazzetta, J.; Law, S.; Biniecki, K. Stability of Commonly Used Antibiotic Solutions in an Elastomeric Infusion Device. Can. J. Hosp. Pharm. 2010, 63, 212-224. [CrossRef] [PubMed]

31. Al Madfai, F.; Zaidi, S.T.R.; Ming, L.C.; Wanandy, T.; Patel, R.P. Physical and Chemical Stability of Ceftaroline in an Elastomeric Infusion Device. Eur. J. Hosp. Pharm. 2018, 25, e115-e119. [CrossRef] [PubMed]

32. Bhattacharya, S.; Parekh, S.; Dedhiya, M. In-Use Stability of Ceftaroline Fosamil in Elastomeric Home Infusion Systems and MINI-BAG Plus Containers. Int. J. Pharm. Compd. 2015, 19, 432-436. [PubMed]

33. Bednar, D.A.; Klutman, N.E.; Henry, D.W.; Fox, J.L.; Strayer, A.H. Stability of Ceftazidime (with Arginine) in an Elastomeric Infusion Device. Am. J. Health Syst. Pharm. 1995, 52, 1912-1914. [CrossRef] [PubMed]

34. Stendal, T.L.; Klem, W.; Tønnesen, H.H.; Kjønniksen, I. Drug Stability and Pyridine Generation in Ceftazidime Injection Stored in an Elastomeric Infusion Device. Am. J. Health Syst. Pharm. 1998, 55, 683-685. [CrossRef]

35. Raby, E.; Naicker, S.; Sime, F.B.; Manning, L.; Wallis, S.C.; Pandey, S.; Roberts, J.A. Ceftolozane-Tazobactam in an Elastomeric Infusion Device for Ambulatory Care: An in Vitro Stability Study. Eur. J. Hosp. Pharm. 2020, 27, e84-e86. [CrossRef]

36. Terracciano, J.; Rhee, E.G.; Walsh, J. Chemical Stability of Ceftolozane/Tazobactam in Polyvinylchloride Bags and Elastomeric Pumps. Curr. Res. Clin. Exp. 2017, 84, 22-25. [CrossRef] [PubMed] 
37. Crandon, J.L.; Sutherland, C.; Nicolau, D.P. Stability of Doripenem in Polyvinyl Chloride Bags and Elastomeric Pumps. Am. J. Health Syst. Pharm. 2010, 67, 1539-1544. [CrossRef] [PubMed]

38. Phipps, D.; Peacock, F.; Smith, L. Stability of Ertapenem in an Elastomeric Infusion Device. Int. J. Pharm. Compd. $2011,15,252$.

39. Foy, F.; Luna, G.; Martinez, J.; Nizich, Z.; Seet, J.; Lie, K.; Sunderland, B.; Czarniak, P. An Investigation of the Stability of Meropenem in Elastomeric Infusion Devices. Drug Des. Dev. 2019, 13, 2655-2665. [CrossRef] [PubMed]

40. Smith, D.L.; Bauer, S.M.; Nicolau, D.P. Stability of Meropenem in Polyvinyl Chloride Bags and an Elastomeric Infusion Device. Am. J. Health Syst Pharm 2004, 61, 1682-1685. [CrossRef]

41. Chen, I.H.; Martin, E.K.; Nicolau, D.P.; Kuti, J.L. Assessment of Meropenem and Vaborbactam Room Temperature and Refrigerated Stability in Polyvinyl Chloride Bags and Elastomeric Devices. Clin. Ther. 2020, 42, 606-613. [CrossRef]

42. Sand, P.; Aladeen, T.; Kirkegaard, P.; LaChance, D.; Slover, C. Chemical Stability of Telavancin in Elastomeric Pumps. Curr. Res. Clin. Exp. 2015, 77, 99-104. [CrossRef]

43. Post, T.E.; Kamerling, I.M.C.; van Rossen, R.C.J.M.; Burggraaf, J.; Stevens, J.; Dijkmans, A.C.; Heijerman, H.G.M.; Touw, D.J.; van Velzen, A.J.; Wilms, E.B. Colistin Methanesulfonate Infusion Solutions Are Stable over Time and Suitable for Home Administration. Eur. J. Hosp. Pharm. 2018, 25, 337-339. [CrossRef] [PubMed]

44. Abdulla, A.; van Leeuwen, R.W.F.; de Vries Schultink, A.H.M.; Koch, B.C.P. Stability of Colistimethate Sodium in a Disposable Elastomeric Infusion Device. Int. J. Pharm. 2015, 486, 367-369. [CrossRef] [PubMed]

45. Tsiouris, M.; Ulmer, M.; Yurcho, J.F.; Hooper, K.L.; Gui, M. Stability and Compatibility of Reconstituted Caspofungin in Select Elastomeric Infusion Devices. Int. J. Pharm. Compd. 2010, 14, 436-439. [PubMed]

46. Harmanjeet, H.; Zaidi, S.T.R.; Ming, L.C.; Wanandy, T.; Patel, R.P. Physicochemical Stability of Voriconazole in Elastomeric Devices. Eur. J. Hosp. Pharm. 2018, 25, e88-e92. [CrossRef]

47. Saillen, L.; Arensdorff, L.; Moulin, E.; Voumard, R.; Cochet, C.; Boillat-Blanco, N.; Gardiol, C.; de Vallière, S. Patient Satisfaction in an Outpatient Parenteral Antimicrobial Therapy (OPAT) Unit Practising Predominantly Self-Administration of Antibiotics with Elastomeric Pumps. Eur. J. Clin. Microbiol. Infect. Dis. 2017, 36, 1387-1392. [CrossRef] [PubMed]

48. Skryabina, E.A.; Dunn, T.S. Disposable Infusion Pumps. Am. J. Health Syst. Pharm. 2006, 63, 1260-1268. [CrossRef]

49. Chung, I.S.; Cho, H.S.; Kim, J.A.; Lee, K.H. The Flow Rate of the Elastomeric Balloon Infusor Is Influenced by the Internal Pressure of the Infusor. J. Korean Med. Sci. 2001, 16, 702-706. [CrossRef]

50. MacKenzie, M.; Rae, N.; Nathwani, D. Outcomes from Global Adult Outpatient Parenteral Antimicrobial Therapy Programmes: A Review of the Last Decade. Int. J. Antimicrob. Agents 2014, 43, 7-16. [CrossRef]

51. Schrank, G.M.; Wright, S.B.; Branch-Elliman, W.; LaSalvia, M.T. A Retrospective Analysis of Adverse Events among Patients Receiving Daptomycin versus Vancomycin during Outpatient Parenteral Antimicrobial Therapy. Infect. Control. Hosp. Epidemiol. 2018, 39, 947-954. [CrossRef] [PubMed]

52. Cervera, C.; Sanroma, P.; González-Ramallo, V.; García de la María, C.; Sanclemente, G.; Sopena, N.; Pajarón, M.; Segado, A.; Mirón, M.; Antón, F.; et al. Safety and Efficacy of Daptomycin in Outpatient Parenteral Antimicrobial Therapy: A Prospective and Multicenter Cohort Study (DAPTODOM Trial). Infect. Dis. 2017, 49, 200-207. [CrossRef]

53. Burnett, Y.J.; Spec, A.; Ahmed, M.M.; Powderly, W.G.; Hamad, Y. Experience with Liposomal Amphotericin B in Outpatient Parenteral Antimicrobial Therapy. Antimicrob. Agents Chemother. 2021, 65, e01876-20. [CrossRef] [PubMed]

54. Gil-Navarro, M.V.; Luque-Marquez, R.; Báez-Gutiérrez, N.; Álvarez-Marín, R.; Navarro-Amuedo, M.D.; Praena-Segovia, J.; Carmona-Caballero, J.M.; Fraile-Ramos, E.; López-Cortés, L.E. Antifungal Treatment Administered in OPAT Programs Is a Safe and Effective Option in Selected Patients. Enferm. Infect. Microbiol. Clin. 2020, 38, 479-484. [CrossRef] [PubMed]

55. Jenkins, A.; Shanu, S.; Jamieson, C.; Santillo, M. Systematic Review of the Stability of Antimicrobial Agents in Elastomeric Devices for Outpatient Parenteral Antimicrobial Therapy Services Based on NHS Yellow Cover Document Standards. Eur. J. Hosp. Pharm. 2021. [CrossRef] [PubMed]

56. Jenkins, A.; Hills, T.; Santillo, M.; Gilchrist, M.; on behalf of the Drug Stability Working Group of the BSAC UK OPAT Initiative. Extended Stability of Antimicrobial Agents in Administration Devices. J. Antimicrob. Chemother. 2017, 72, dkw556. [CrossRef] [PubMed]

57. Diamantis, S.; Dawudi, Y.; Cassard, B.; Longuet, P.; Lesprit, P.; Gauzit, R. Home Intravenous Antibiotherapy and the Proper Use of Elastomeric Pumps: Systematic Review of the Literature and Proposals for Improved Use. Infect. Dis. Now 2021, 51, 39-49. [CrossRef]

58. Welch, V.; Petticrew, M.; Tugwell, P.; Moher, D.; O’Neill, J.; Waters, E.; White, H.; PRISMA-Equity Bellagio group. PRISMA-Equity 2012 Extension: Reporting Guidelines for Systematic Reviews with a Focus on Health Equity. PLoS Med. 2012, 9, e1001333. [CrossRef] [PubMed] 